\title{
Stimulus fractionation by interocular suppression
}

\author{
Asieh Zadbood ${ }^{1}$, Sang-Hun Lee ${ }^{1}$ and Randolph Blake ${ }^{1,2 *}$ \\ Department of Brain and Cognitive Sciences, Seoul National University, Seoul, South Korea \\ ${ }^{2}$ Department of Psychology, Vanderbilt Vision Research Center, Center for Cognitive and Integrative Cognitive Neuroscience, Vanderbilt University, Nashville, TN, \\ USA
}

Edited by:

Theofanis Panagiotaropoulos, Max Planck Institute for Biological

Cybernetics, Germany

Reviewed by:

Erich Schröger, University of Leipzig, Germany

Sheng He, University of Minnesota, USA

\section{*Correspondence:}

Randolph Blake, Department of Psychology, Vanderbilt University, 111 21 st Avenue South, 301 Wilson Hall, Nashville, TN 37240, USA.

e-mail: randolph.blake@vanderbilt.edu
Can human observers distinguish physical removal of a visible stimulus from phenomenal suppression of that stimulus during binocular rivalry? As so often happens, simple questions produce complex answers, and that is the case in the study reported here. Using continuous flash suppression to produce binocular rivalry, we were able to identify stimulus conditions where most - but not all - people utterly fail to distinguish physical from phenomenal stimulus removal, although we can be certain that those two equivalent perceptual states are accompanied by distinct neural events. More interestingly, we find subtle variants of the task where distinguishing the two states is trivially easy, even for people who utterly fail under the original conditions. We found that stimulus features are differentially vulnerable to suppression. Observers are able to be aware of existence/removal of some stimulus attributes (flicker) but not others (orientation), implying that interocular suppression breaks down the unitary awareness of integrated features belonging to a visual object. These findings raise questions about the unitary nature of awareness and, also, place qualifications on the utility of binocular rivalry as a tool for studying the neural concomitants of conscious visual awareness.

Keywords: awareness, interocular suppression, continuous flash suppression, temporal modulation, orientation, feature-selectivity

\section{INTRODUCTION}

Binocular rivalry fascinates us for several reasons. First, it starkly highlights the brain's strategy for dealing with ambiguous or conflicting visual information. Indeed, some believe the processes revealed during rivalry are operating at all times to resolve ambiguities inherent in the optical input during everyday vision (e.g., Hohwy et al., 2008; Sterzer et al., 2009). Second, alternations in dominance during binocular rivalry imply that conflict resolution is unstable - perception does not settle upon a single, dominant interpretation but, instead, fluctuates over time unpredictably (e.g., Levelt, 1965; Fox and Herrmann, 1967; Brascamp et al., 2005). And third, during rivalry a complex, ordinarily visible stimulus can disappear from visual awareness for seconds at a time even though that stimulus remains imaged on the retina, a remarkable act of disappearance that has been dubbed psychophysical magic (Kim and Blake, 2005). This profound, intermittent dissociation between physical stimulation and perceptual experience affords a paradigmatic case for the study of visual awareness (Crick and Koch, 2003), and the search for fluctuations in neural activity correlated with the intermittent disappearance of a stimulus has been underway for years now (Tong et al., 2006). In this paper, we focus on this third aspect of rivalry, i.e., the temporary invisibility of a stimulus.

Our question is quite simple: can we sense the physical removal of a stimulus that has already been rendered invisible owing to interocular suppression? Perceptually speaking, a suppressed stimulus temporarily ceases to exist, and the "out of sight" quality of such a stimulus naturally leads one to inquire about the extent to which such a stimulus is also "out of mind." In terms of neural events, the two circumstances are surely different. Specifically, physical removal of a stimulus has ramifications beginning at the very earliest stages of vision in the retina; removal of a stimulus from awareness during rivalry, however, does not impact retinal processing except for subtle influences on oculomotor responses (Lorber et al., 1965; Sabrin and Kertesz, 1980). Moreover, physical removal of a stimulus produces larger, more widespread changes in cortical neural activity than does phenomenal removal owing to binocular rivalry (Leopold and Logothetis, 1996; Polonsky et al., 2000; Sterzer et al., 2008). Finally, we know that a stimulus outside of awareness, because it is still present, can generate visual aftereffects arising at different stages of visual processing (Blake and $\mathrm{He}$, 2005), and we know that other aspects of visual information processing associated with that stimulus survive suppression albeit often with reduced effectiveness (Lin and $\mathrm{He}, 2009$ ). But these psychophysical results provide indirect measures of the residual effectiveness of a suppressed stimulus, serving much the same role as footprints in the sand that hint at the presence of an unseen person. For our question we are seeking more than footprints: we want to know whether people can sense the physical removal of a stimulus that has already been perceptually erased from awareness during rivalry. Answering this question could shed light on the nature of suppression and, by extension, on the often-stated assertion that binocular rivalry provides an effective means for studying the neural correlates of consciousness (e.g., van Ee, 2009; Alais et al., 2010).

Here is how we have pursued the question. Using continuous flash suppression (CFS) to create binocular rivalry (Tsuchiya and Koch, 2005), we presented a dynamic Mondrian to one eye and a 
circular gabor pattern to the other eye. While the gabor pattern was suppressed, we removed either the top half or the bottomhalf of the pattern and required observers to judge which half was removed, top or bottom (two-alternative spatial forced-choice). We reasoned that if suppression of the gabor pattern is equivalent to physically removing it, it should be impossible to judge which half is physically removed when the pattern is suppressed (because, perceptually speaking, both halves are already gone). By way of preview, this simple question led to the realization that suppression does not operate in a wholesale fashion on a stimulus but, instead, selectively impacts certain features of the stimulus. This finding squares with earlier results obtained using different techniques, and it reveals that phenomenal suppression is quite different from physical absence.

\section{METHODS}

\section{EXPERIMENT 1}

A total of 16 participants ( 6 men), including 2 of authors (Asieh Zadbood and Randolph Blake), participated in the first experiment (mean age $\sim 28$ years). Eleven of them were naïve and had no previous experience in psychophysical experiments nor any familiarity with binocular rivalry. All had normal or corrected to normal vision, and each gave written consent to procedures as approved by the IRB office at Seoul National University.
Observers viewed the stimuli on a CRT monitor $(1024 \times 768$ resolution, $60 \mathrm{~Hz}$ ) through a mirror stereoscope attached to a head/chin rest. The distance between eyes and display was $71 \mathrm{~cm}$, and the head/chin rest stabilized head position and viewing distance. All the experiments were programmed using MATLAB, ver. 7.4 and Psychtoolbox, ver. 3 (Brainard, 1997; Pelli, 1997).

Unless otherwise specified, all experiments involved presentation of a CFS display to one eye and a Gabor patch comprising horizontal contours to the other eye (Figure 1). For the CFS display we used grayscale Mondrian patterns $\left(4.34^{\circ} \times 4.34^{\circ}\right)$ normalized to $70 \%$ contrast (root mean square). Each Mondrian frame comprised overlapping rectangles of variable dimensions and variable luminance. A new Mondrian image was presented every $100 \mathrm{~ms}$ $(10 \mathrm{~Hz})$ throughout the duration of each trial. As noted by others (e.g., Tsuchiya and Koch, 2005), this dynamic, richly contoured display is a potent generator of interocular suppression, as evidenced by its long durations of dominance when paired with a rival stimulus to the other eye.

The target stimulus viewed by the other eye was a counter-phase flickering, horizontal Gabor patch $\left(3.1^{\circ} \times 3.1^{\circ}\right.$ grating + Gaussian envelope $\mathrm{SD}=0.95^{\circ}$ ) the spatial frequency of which was $1.3 \mathrm{c} /$ degree; the pattern flickered in counterphase (Levinson and Sekuler, 1975) at $1 \mathrm{~Hz}$. The spatial position of the Gabor patch was precisely situated so that the pattern's zero crossing was located at

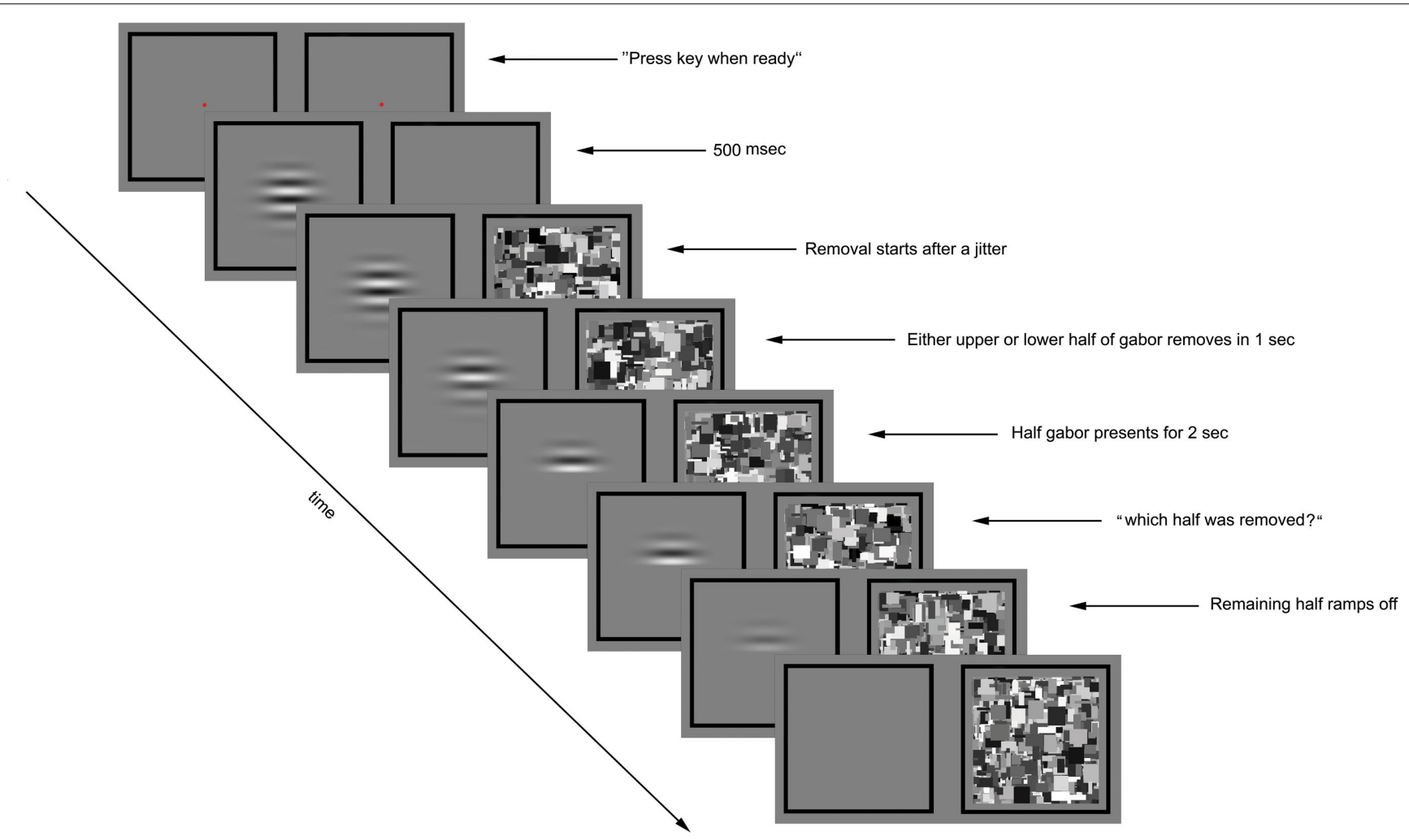

FIGURE 1 | Experimental paradigm for preview condition of

Experiment 1. The trial was initiated by the observer's key press. A flickering gabor was presented to one eye and blank gray square to the other eye. After $500 \mathrm{~ms}$ the Mondrian images were displayed to the other eye at $10 \mathrm{~Hz}$. Following a variable time period $(0,200$, or $400 \mathrm{~ms})$ the contrast of either the upper or lower half of the gabor was gradually ramped to zero over a 1000-ms period. The other half remained unchanged for $2 \mathrm{~s}$ before the prompt screen asked about the half removal side, at which time the remaining half of the gabor was removed over a $300 \mathrm{~ms}$ period. The CFS was displayed to the other until the observer made his/her response. 
the exact middle of the display during all phases of the sinusoidal flicker cycle. Four different contrast values $(0.05,0.1,0.2,0.4)$ were used in this first experiment.

To achieve and maintain stable binocular alignment, a test session started with presentation of two black square frames $\left(4.94^{\circ} \times 4.94^{\circ}\right)$ with a red circle $\left(0.25^{\circ} \times 0.25^{\circ}\right)$ in the middle as the fixation point; the observer started each session by adjusting the mirrors of the stereoscope using the cover/uncover test, pressing a key when refixation eye movements associated with repetitive monocular viewing of the two half-images had been eliminated.

During each test session, two exposure conditions were randomly intermixed, one called preview and the other called postview. On preview trials, the Gabor patch was presented to one eye followed $500 \mathrm{~ms}$ later by presentation of the CFS animation to the other eye (Figure 1). On post-view trials, the CFS animation was presented first followed $500 \mathrm{~ms}$ later by presentation of the Gabor patch, whose contrast was ramped on to its given value for that trial over $500 \mathrm{~ms}$. On all trials, the upper or the lower half of the Gabor patch was gradually removed; because the contours were horizontal and the pattern was centered at its zero crossing, removal of half of the Gabor produced no change in average luminance. On preview trials (Gabor preceding the Mondrian), removal of the upper or the lower half of the Gabor patch occurred 0 (simultaneous), 200, or $400 \mathrm{~ms}$ after presentation of the Mondrian, with the delay determined randomly for each trial. On post-view trials (Gabor following the Mondrian), removal of half of the Gabor patch occurred either 500, 700, or $900 \mathrm{~ms}$ after appearance of the Gabor, with that delay value randomly determined for each trial.

The rest of timeline was same for preview and post-view conditions: the removal was completed gradually in $1000 \mathrm{~ms}$ and the remaining half of the Gabor was not removed until $2000 \mathrm{~ms}$ later. The CFS animation was shown to the other eye during the whole period of trial. Then a screen prompt instructed the observer to indicate by button press which half of the Gabor had been removed, guessing if necessary. At the same time the remaining portion of the Gabor patch was removed and the CFS animation remained on during this response period, to mask any lingering afterimage associated with removal of the Gabor patch. Observers were asked to respond as quickly as possible, and they were told that the entire Gabor patch would be gone at the time they made their response so they should judge based on what they experienced during the trial not what they experienced at the time of the screen prompt.

All timing conditions, preview and post-view trials, stimulus removal location (upper vs. lower) and stimulus eye assignments were randomized and counterbalanced across trials.

Each condition (preview vs. post-view for each of four contrast values) was repeated 108 times, and each observer completed 864 trials in this task.

\section{EXPERIMENT 2}

\section{Part one}

Fifteen observers from the first experiment participated in this experiment (including the two authors).

The CFS display and timeline were the same as in the previous experiment. The Gabor patch was the same, too, except that its contrast was 0.4 and it had one of two counter-phase flicker rates,
1 or $5 \mathrm{~Hz}$. The Gabor patch was always presented first (preview mode), and either the upper or the lower half of the Gabor was removed with the same timing used in the first experiment. The observer's task was the same, and there were 108 trials per each condition and a total of 216 trials for each observer.

\section{Part two}

Eight of the participants in part one also completed the second part of Experiment 2.

The stimulus was a Gabor patch with the same properties explained above in first part but it was not flickering. The task and timing conditions were the same. Each observer completed 108 trials.

\section{EXPERIMENT 3}

Six observers participated in this experiment. Target stimuli were two gabor patches $\left(2^{\circ} \times 2^{\circ}\right.$ gratings smoothed by a Gaussian envelope with $\mathrm{SD}=0.57^{\circ}$ ). These two gabors were centered immediately above and below a central fixation point (Figure 2 ), and their contours were diagonally oriented such that one was $45^{\circ}$ and the other was $135^{\circ}$; the orientation/location pairing was random over trials. The spatial frequency of each gabor patch was $2 \mathrm{c} /$ degree, and on each trial both flickered in counterphase at one of four temporal frequencies: $0.5,1,2$, or $5 \mathrm{~Hz}$. The contrast values of the two gabor patches were identical and were established for each observer based on performance in Experiment 1 and in pilot testing for Experiment 3. The CFS display again comprised a sequence of grayscale Mondrian patterns (70\% contrast), with a new image presented every $100 \mathrm{~ms}$. For this experiment, the CFS was enlarged to $5.5^{\circ} \times 5.5^{\circ}$, to match the larger size of the rival stimuli presented to the other eye.

The session began with presentation of a pair of binocular fusion frames, one viewed by the left eye and the other by the right eye. Each frame consisted of a thick black square $\left(6.2^{\circ} \times 6.2^{\circ}\right)$ with small, central fixation mark. Once the observer had adjusted the mirrors of the stereoscope to achieve stable binocular alignment of these fusion frames, he/she pressed a key that triggered presentation of the CFS display to one eye or the other. Then, $500 \mathrm{~ms}$ after presentation of CFS, the pair of gabor patches was ramped on, creating the post-view condition described earlier. Then after a short interval lasting either 500, 700, or $900 \mathrm{~ms}$, one of the two gabor patches was gradually removed over a $1000-\mathrm{ms}$ period time. The other gabor remained present for two more seconds, at which time its contrast dropped to zero and the observer was cued to make two judgments: (1) the orientation of the initially removed grating and

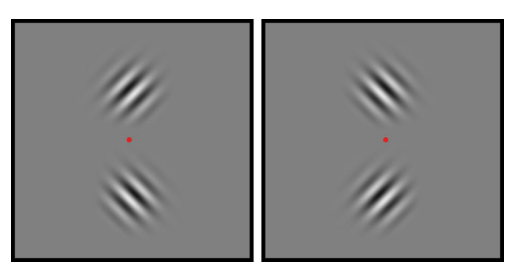

FIGURE 2 | Target stimuli for Experiment 3. Two gabor patches, one tilted $45^{\circ}$ clockwise and the other tilted $45^{\circ}$ counterclockwise, were located above and below the central fixation point. 
(2) the location (above vs. below fixation) of the initially removed grating. During the response period, the other eye continued to view the CFS display until both responses were made. All trialrelated variables - eye receiving the pair of gabor patches, orientation/location of the gabor patches and temporal frequency - were randomized with the stipulation that all combinations be presented an equal number of trials. Observers completed 216 trials for each of the four temporal frequency values.

\section{RESULTS}

\section{RATIONALE OF THE PRESENT STUDY}

Before presenting our results, we start with a description of the rationale that guided our design of these experiments. In principle, the most straightforward way to answer the question of the equivalence of phenomenal and physical absence is simply to determine whether or not observers can tell if a stimulus has been presented to one eye (on some trials it is, on other trials it is not), with the other eye always viewing the dynamic Mondrian. We rejected this simple approach, however, because it does not capture the essence of "absence" that we are interested in: the removal of a stimulus from phenomenal awareness because of interocular suppression. We wanted to know, in other words, whether physical removal is distinguishable from phenomenological removal, and that means presenting a stimulus on every trial and removing it on some trials but not others.

With that in mind, we initially tried a "yes/no" procedure whereby a gabor patch was presented to one eye at the same time as the CFS Mondrian was presented to the other eye. On half of the trials the entire gabor patch faded off after 1-2 s, leaving just the CFS display; on the other trials, the gabor patch remained present. Knowing that afterimages can mimic real patterns in rivalry (Wade, 1974), we flickered the gabor patch on all trials to preclude induction of a residual afterimage on those trials when the pattern was removed (Gilroy and Blake, 2005). Observers were cued with an instruction to guess whether or not the gabor patch was removed. Observers characterized the task as impossible, and the $d^{\prime}$ values confirmed that observers could not tell on which trials it was removed. While implying that awareness of absence is not noticeable during absence of awareness, we were unwilling to accept this as a definitive answer to our question. Instead, we elected to employ a forced-choice technique wherein either the top- or bottom-half of the gabor patch disappeared on every trial and observers made a forced-choice judgment about which half was removed, guessing if necessary. We moved to this procedure knowing that forced-choice testing typically produces better performance than does the yes/no procedure (e.g., Macmillan and Creelman, 1991; Kroll et al., 2002), a textbook example being the above-chance localization performance that can be obtained in the blind hemifield of at least some individuals with unilateral occipital lobe lesions (e.g., Weiskrantz, 1990). In addition to the more sensitive nature of 2AFC task, this new design differs from the original one in a subtle but important way: removing only half of the suppressed stimulus, not all of it, provides a potential reference frame in the region spatially contiguous with the region of interest, thereby allowing observers to sense subtle differences by directly comparing physical absence and phenomenal absence.
For this 2AFC experiment, we made several strategic decisions concerning the test stimulus that would be partially removed. First, we decided to test two methods for presenting the gabor patch, one involving its presentation shortly before appearance of the CFS Mondrian and the other involving its presentation shortly after appearance of the CFS Mondrian. We did this reasoning that the former would insure that a neural representation of the gabor patch would be formed before the onset of suppression induced by the Mondrian; we could not be sure this would happen when gabor patch followed the Mondrian. Second, we chose this particular range of gabor patch contrasts because, with these contrasts, it was trivially easy to see which half was removed when the monocularly presented pattern was dominant owing to the absence of the Mondrian; every participant performed flawlessly on this condition. What happened, however, when the gabor patch was suppressed by CFS?

\section{EXPERIMENTS 1 AND 2}

Results are shown in Figure 3, which plots percent-correct (chance $=50 \%$ ) as a function of the contrast of the gabor patch. We have pooled results across each observer's left and right eyes, as separate analyses contingent on the eye receiving the gabor patch disclosed no systematic performance differences $[F(1,15)=0.96$, $P=0.34]$.Several features are obvious from these psychometric functions. First, some, but not all, observers found the task impossible regardless of contrast. Those whose performance did climb above the chance level ( $>58 \%$ correct, based on the binomial distribution with 108 trials/condition) generally showed improved performance primarily at the higher contrasts. Among observers who were at least partially successful, there was no obvious tendency for them to perform better when the gabor patch appeared before being suppressed $[F(1,9)=0.8, P=0.39]$ - evidently the neural representation of the gabor patch is attenuated equivalently whether or not its initial neural representation is established prior to succumbing to interocular suppression.

Observers who were able to perform the task offered a revealing account of the cue they relied on: they described seeing faint ripples within either the top- or bottom-half of the dynamic Mondrian pattern a few moments after onset of a trial, and the location of these ripples in either the top or the bottom portion of the Mondrian informed their forced-choice judgment. As is obvious from Figure 3, these ripples were inconspicuous at low contrasts. Once these ripples were discovered within the CFS, it was possible to discern that they were also faintly visible when the entire gabor patch was present, although they were much more conspicuous when half of the gabor patch was removed. The first author was one of those for whom the ripples were noticeable, and she quickly understood that the ripples coincided with the counterphase flicker of the gabor patch. To confirm that this rippling cue was indeed tied to the flicker, we retested a subset of observers using a non-flickering, stationary gabor patch with highest contrast, and performance was at or near chance for all observers (mean $=0.6, \mathrm{SD}=0.08$ ) including those whose performance was previously high.

These results - and the anecdotal descriptions of the residual flicker signal associated with the suppressed gabor patch - prompted us to retest observers at two different rates of 


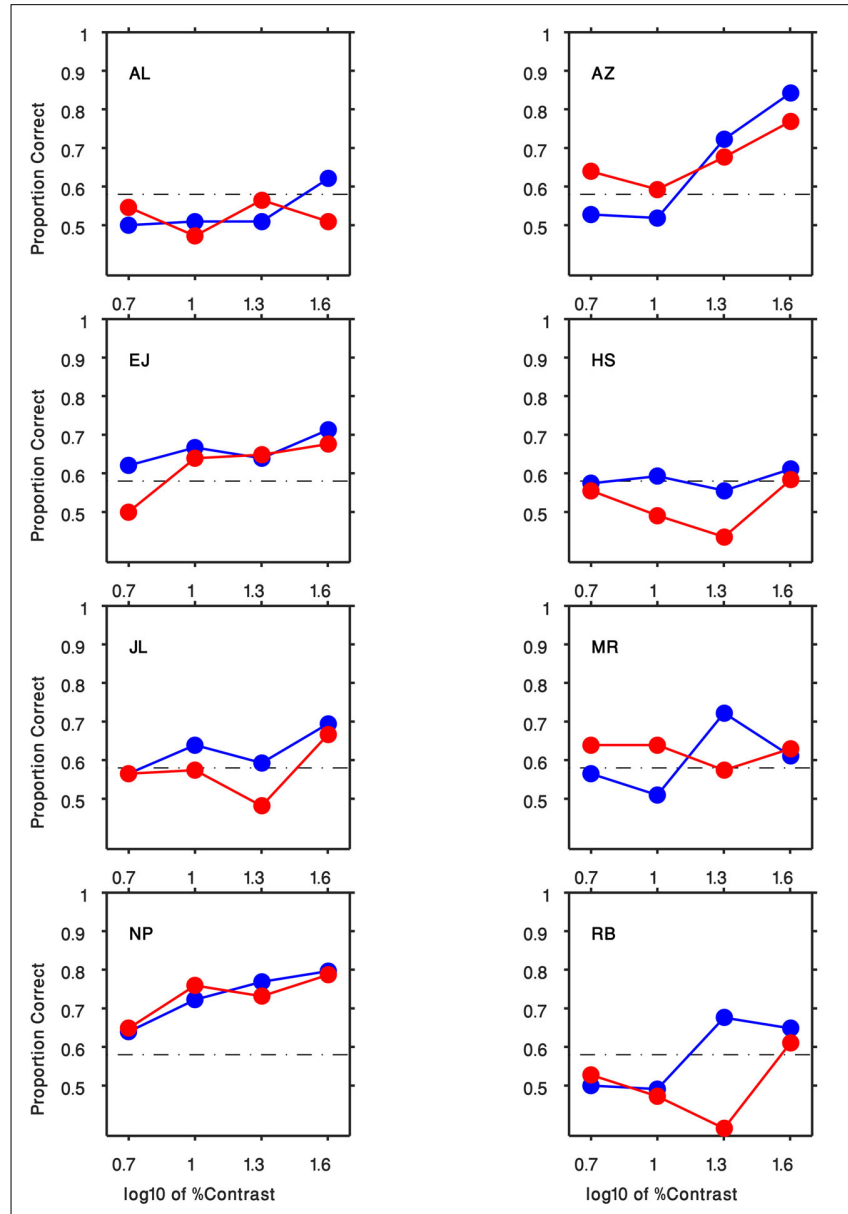

FIGURE 3 | Results of Experiment 1 for all 16 participants. Each panel indicates the result of an observer (initials indicated in the top left corner). The preview condition is shown in blue circles and the post-view condition is shown in red circles. Horizontal axis is the $\log _{10}$ of the gabor patch percent
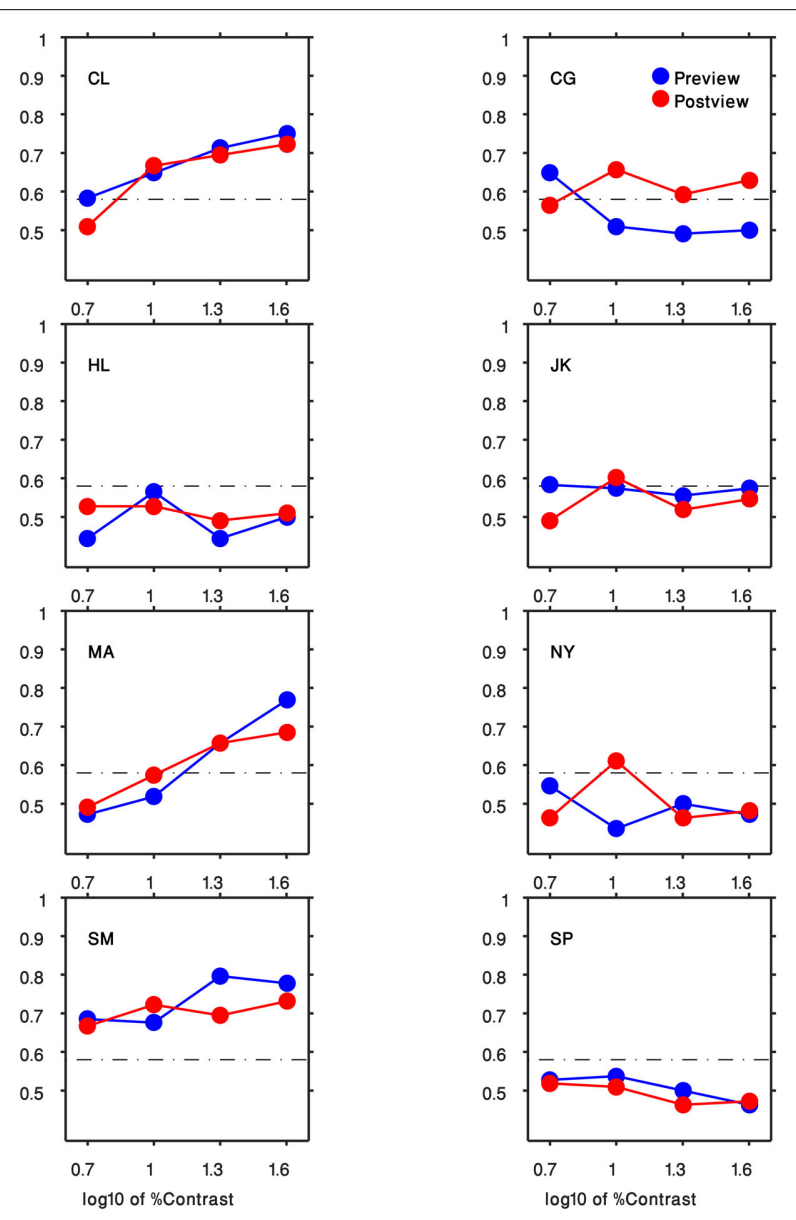

contrast $(5,10,20,40)$. Vertical axis plots the proportion of correct responses. The black dashed lines denote the level of performance significantly above-chance level (58\% correct, based on the binomial distribution with 108 trials/condition). counter-phase flicker, 1 and $5 \mathrm{~Hz}$. For these measurements we tested using the preview procedure only (i.e., gabor patch introduced before the Mondrian), and we tested at the high contrast only, 40\%. Results from those conditions are plotted in Figure 4A, where it can be seen that observers performed much better at $5 \mathrm{~Hz}$, a flicker rate where the faint ripples were now sufficiently strong to make the disappearance cue more conspicuous $[t(14)=-7.43$, $P<0.0001]$. The third author was retested, this time including intermediate flicker values, and his results confirm that this cue becomes increasingly more salient with flicker rate (Figure 4B). We have not yet tried to identify the upper flicker rate beyond which this cue begins to weaken, but based on our knowledge of the effect of temporal frequency on contrast sensitivity (Robson, 1966) there is bound to be a point at which one begins to lose sensitivity to this cue.

So, it appears that interocular inhibition induced by a dynamic Mondrian can effectively erase from awareness the form information associated with our test stimulus while exerting a less robust suppression effect on the temporal information carried by that stimulus.

\section{EXPERIMENT 3}

The ripples within the CFS that betray the location of the removed portion of a gabor patch themselves convey a vague sense of form, in the same way that waves in a body of water portray form in virtue of their spatial extension. In the parlance of contemporary vision science, we would characterize such waves as second-order, or non-Fourier, patterns defined by temporal modulation, not by luminance (Lin and Wilson, 1996). In this third experiment, we asked whether interocular suppression induced by CFS exerts differential effects on absence of awareness of this second-order form information compared to absence of awareness of stimulus location disclosed by temporal modulation. To pursue this question, we exploited the increasing salience of higher rates of flicker as a cue for location (recall Figure 4). Does identification of second-order contour orientation covary with increasingly improved location performance? To find out, we modified our previous task to turn it into a $2 \times 2$ forced-choice task that assesses performance on two tasks concurrently (Nachmias and Weber, 1975; Watson and Robson, 1981). 


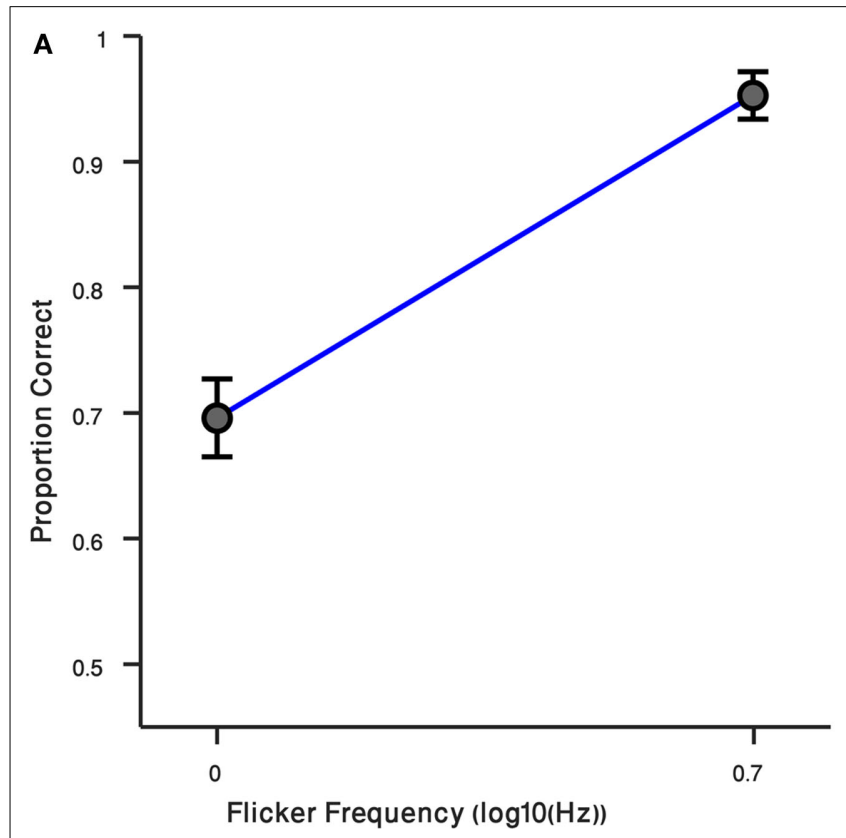

FIGURE 4 | Result of Experiment 4. (A) Performance of observers in two flicker frequency conditions ( 1 and $5 \mathrm{~Hz}$ ). Error bars indicate \pm 1 SEM. (B) Result of Randolph Blake in four flicker frequency conditions $(0.5,1,2,5 \mathrm{~Hz})$

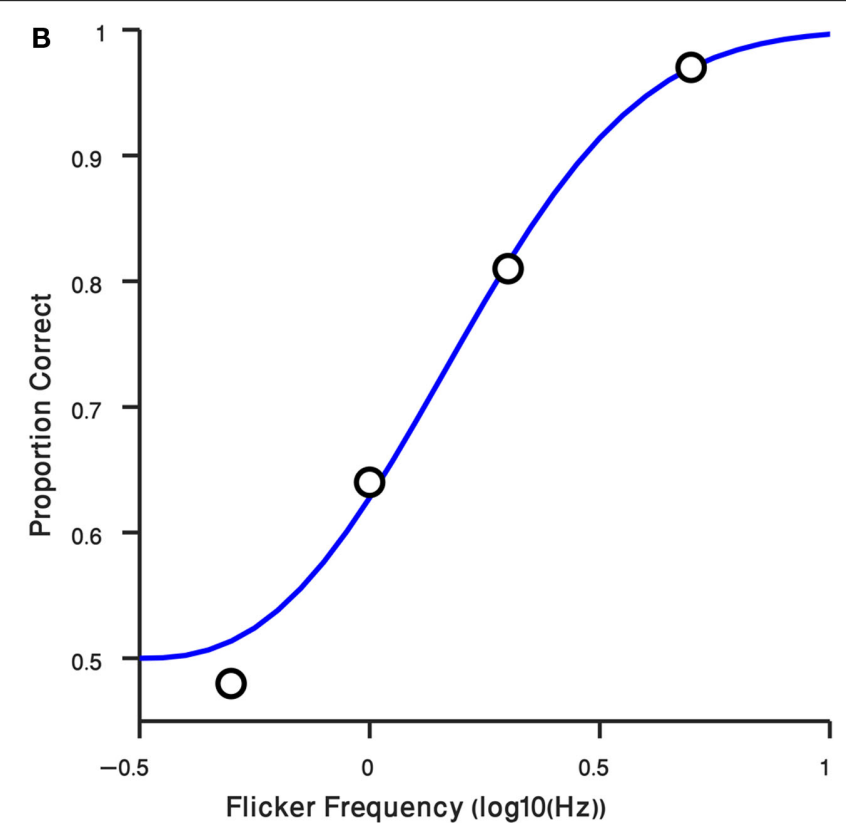

are shown in black circles. The blue curve is a Weibull function fitted to data. Horizontal axis is the $\log _{10}$ of flicker frequency. Vertical axis plots the proportion of correct responses.
In this third experiment, we modified the display presented to one eye so that it contained two flickering gabor patches situated above and below a central fixation point (Figure 2 ), with one comprising contours oriented clockwise and the other comprising contours oriented anticlockwise; the other eye viewed the CFS display. From trial to trial the orientation/location pairings of the gabor patches were randomized, as was the eye receiving the pair of gabor patches. On each trial the observer judged the orientation and the location of the gabor patch that was removed $2 \mathrm{~s}$ before removal of the second gabor patch.

For each observer these dual 2AFC tasks produced two sets of data, one associated with identification of the orientation of the initially removed pattern and the other associated with identification of the location of the initially removed pattern. For each observer we analyzed those two sets of results in the following way. We first used a maximum likelihood procedure to fit a Weibull psychometric function to each data set (examples of the two extremes in performance among the six observers are shown in the upper panels of Figure 5). Using those best-fitting psychometric functions, we next applied a parametric bootstrapping technique (Wichmann and Hill, 2001) to estimate the $95 \%$ confidence intervals of the flicker frequency value associated with $65 \%$ correct performance on the location task and on the second-order orientation task. If performance on those two tasks were perfectly yoked, we would expect those estimated threshold values to be statistically indistinguishable.

The lower left scatterplot in Figure 5 reveals that the threshold for judging the location of the removed stimulus was consistently higher than the threshold for judging the orientation of that same stimulus. For all observers, in other words, there were trials on which they could perceive the location of the removed pattern but could not perceive its orientation. Once again, observers described using a process of elimination to perform the location judgment: either the upper or the lower region of the CFS display ceased to exhibit that vague sense of rippling produced by the temporal modulation of the gabor patch, enabling the observer to deduce whether the initially removed gabor was the one located above or below fixation. Evidently this cue was less salient for perceiving the orientation of that initially removed pattern (or, for that matter, less salient for specifying the orientation of the remaining gabor patch which, by process of elimination, would enable observers to deduce the orientation of the initially removed pattern).

This dissociation between absence of awareness of stimulus orientation and stimulus location was further evidenced by an analysis of performance of the orientation task contingent on correct performance on the location task. Specifically, for each observer we computed the conditional probability of correct orientation judgment given a correct location judgment, $P\left(C_{\mathrm{O}} / C_{\mathrm{L}}\right)$, and the conditional probability of a correct location judgment given a correct orientation judgment, $P\left(C_{\mathrm{L}} / C_{\mathrm{O}}\right)$. For all six observers, $P\left(C_{\mathrm{O}} / C_{\mathrm{L}}\right)$ was lower than $P\left(C_{\mathrm{L}} / C_{\mathrm{O}}\right)$, again pointing to a dissociation between performance on the two tasks (lower right scatter plot in Figure 5).

\section{DISCUSSION}

This study was initially conceptualized as an attempt to learn whether awareness of absence is distinguishable from absence of awareness. Based on what we have learned from our three experiments, we have no doubt that it is possible to create conditions where observers cannot notice the physical removal of a stimulus that was already erased from awareness by interocular suppression. But in the course of performing these experiments, we stumbled 

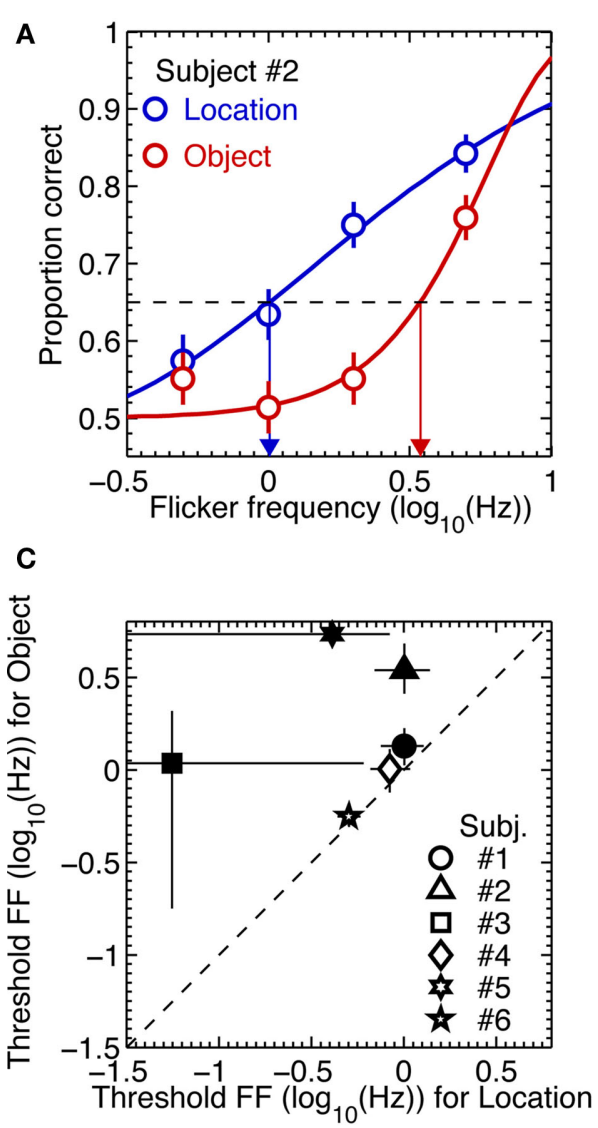

FIGURE $\mathbf{5}$ | Results of Experiment 3. (A,B) Example psychometric function fits to the data from two most extreme observers. The blue and red symbols are data from the location and object tasks, respectively. The thick lines are Weibull functions fitted to the data. The blue and red arrows indicate $65 \%$ (the broken horizontal lines) threshold flicker frequencies for the location and object tasks, respectively. The error bars are the standard errors of proportion correct, estimated from binomial distributions. (C) Threshold flicker frequencies from the location task are plotted against those from the object task. The different symbols represent different observers. The

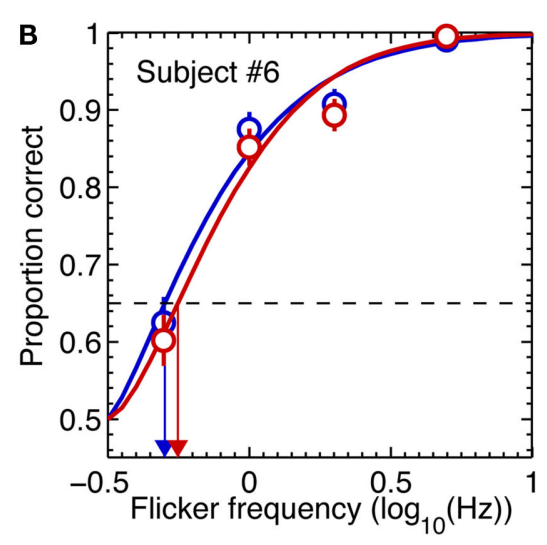

D

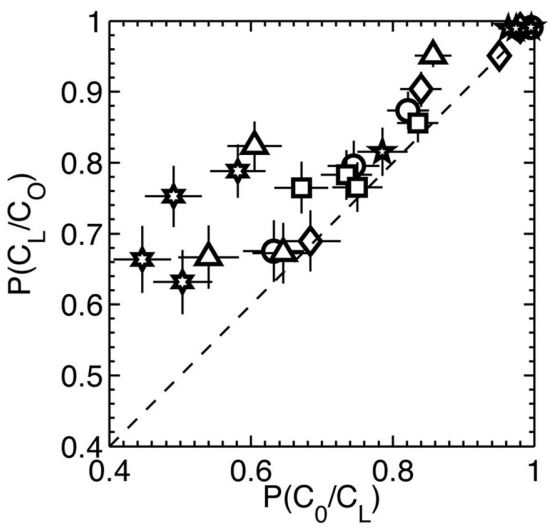

statistically significant differences in threshold between the two tasks are indicated by the solid symbols. The error bars are $95 \%$ confidence intervals, estimated from 2000 parametric bootstrap samples. (D) The conditional probability of being correct in the object task given correct location judgment are plotted against the conditional probability of being correct in the location task given correct object judgment for each of flicker frequency and for each subject. The different symbols represent different observers. The error bars are the standard errors of proportion correct, estimated from binomial distributions.

integration of the two flicker rates even when they were consciously aware of only one of the two forms. Flicker and form information, in other words, were dissociated in perception (see He et al., 2005, for more on this dissociation and its possible relation to the parvo- and magnocellular pathways). Flicker and form are not the only stimulus qualities that can be dissociated during suppression. Hong and Blake (2009) noted that, "the color of an object, suppressed from awareness during CFS, could nonetheless be experienced as a diffuse, somewhat faint cloud appearing transparently on the grayscale rectangles forming the Mondrian patterns. This impression of color did not seem to be a surface property of the Mondrian itself but, instead, to be a transparent overlay with no defined shape. (p. 403)." Hong and Blake documented this dissociation in a series of experiments, including one that involved monocular presentation of colored, drifting gratings. Results from that latter condition showed that increasing the speed of motion of a colored grating made its color more difficult to identify during suppression but made its direction of motion 
easier to identify. This latter finding, of course, dovetails with the frequency-dependent modulation of detection of absence found in our Experiment 2. (In an email exchange with one of the authors (Randolph Blake), Bahador Bahrami, University College, London, described a similar phenomenon when using CFS to suppress a drifting grating, writing that the contrast polarity of the grating was invisible but the drift was still evident "like a mirage in a desert.")

Putting these various findings together, one is led to the conclusion that neural information associated with some aspects of a stimulus are less susceptible to interocular suppression than are other aspects of that stimulus. Interocular suppression thus can operate like the chemical process of fractional distillation, separating the qualia comprising conscious visual awareness from the objects or events themselves. The idea that the contents of awareness can be fractionated by suppression is intriguing within the context of the view that awareness is a constructive process that operates to unify/integrate sensory information (e.g., Baars, 2005; Mudrik et al., 2011). While not contradicting this view, fractionation of awareness by suppression implies that perceptually bound features can become temporarily unbound

\section{REFERENCES}

Alais, D., Cass, J., O'Shea, R. P., and Blake, R. (2010). Visual sensitivity underlying changes in visual consciousness. Curr. Biol. 20, 1362-1367.

Andrews, T., and Blakemore, C. (2002). Integration of motion information during binocular rivalry. Vision Res. 42, 301-309.

Andrews, T. J., and Blakemore, C. (1999). Form and motion haveindependent access to consciousness. Nat. Neurosci. 2, 405-406.

Baars, B. J. (2005). Global workspace theory of consciousness: toward a cognitive neuroscience of human experience. Prog. Brain Res. 150, 45-53.

Blake, R., and He, S. (2005). "Visual adaptation as a tool for studying the neural correlates of conscious visual awareness," in Fitting the Mind to the World, eds C. Clifford and G. Rhodes (New York, NY: Oxford University Press), 281-307.

Brainard, D. H. (1997). The Psychophysics Toolbox. Spat. Vis. 10, 443-446.

Brascamp, J. W., van Ee, R., Pestman, W. R., and van der Berg, A. V. (2005). Distributions of alternation rates in various forms of bistable perception. J. Vis. 5, 287-298.

Carlson, T. A., and He, S. (2000). Visible binocular beats from invisible monocular stimuli during binocular rivalry. Curr. Biol. 10, 1055-1058.

Crick, F., and Koch, C. (2003). A framework for consciousness. Nat. Neurosci. 6, 119-126.
Fox, R., and Herrmann, J. (1967). Stochastic properties of binocular rivalry alternations. Percept. Psychophys. 2, 432-436.

Gilroy, L. A., and Blake, R. (2005). The interaction between binocular rivalry and negative afterimages. Curr. Biol. 15, 1740-1744.

He, S., Carlson, T. A., and Chen, X. (2005). "Parallel pathways and temporal dynamics in binocular rivalry," in Binocular Rivalry and Perceptual Ambiguity, eds D. Alais and R. Blake (Boston, MA: MIT Press), 81-100.

Hohwy, J., Roepstorff, A., and Friston, K. (2008). Predictive coding explains binocular rivalry: an epistemological review. Cognition 108, 687-701.

Holopigian, K. (1989). Clinical suppression and binocular rivalry suppression: the effects of stimulus strength on the depth of suppression. Vision Res. 29, 1325-1333.

Hong, S. W., and Blake, R. (2009). Interocular suppression differentially affects chromatic an achromatic mechanisms. Atten. Percept. Psychophys. 71, 405-411.

Kim, C. Y., and Blake, R. (2005). Psychophysical magic: rendering the visible "invisible". Trends Cogn. Sci. (Regul. Ed.) 9, 381-388.

Kroll, N. E., Yonelinas, A. P., Dobbins, I. G., and Frederick, C. M. (2002). Separating sensitivity from response bias: implications of comparisons of yes-no and forced-choice tests for models and measures of recognition memory. J. Exp. Psychol. Gen. 131, 241-254. without losing access to consciousness. That being said, we are reluctant to push this idea too far, for the very notion of qualia is highly controversial as evidenced by the lively debates over this notion among philosophers (see the Wikipedia entry for qualia http://en.wikipedia.org/wiki/Qualia to see what we mean).

In the vision literature, people often speak of the depth of suppression when referring to binocular rivalry (e.g., Nguyen et al., 2001) or to interocular suppression associated with strabismic amblyopia (Holopigian, 1989). Our findings suggest that it may be useful to think about awareness as having depth, in that awareness can penetrate to different levels depending on the composition of the stimulus and, perhaps, the nature of the task being performed. We are currently exploring whether the depth of awareness is dependent on task demands and whether it can be modified by practice and, if so, the extent to which such modification generalizes to other tasks or categories of stimuli.

\section{ACKNOWLEDGMENTS}

Supported by the WCU program through the National Research Foundation of Korea funded by the Ministry of Education, Science, and Technology (R31-10089).

Leopold, D. A., and Logothetis, N. K. (1996). Activity changes in early visual cortex reflect monkeys' percepts during binocular rivalry. Nature 379, 549-553.

Levelt, W. J. M. (1965). On Binocular Rivalry. Soesterberg: Institute for Perception RVO-TNO.

Levinson, E., and Sekuler, R. (1975) The independence of channels in human vision selective for direction of movement. J. Physiol. (Lond.) 250, 347-366.

Lin, L. M., and Wilson, H. R. (1996). Fourier and non-Fourier pattern discrimination compared. Vision Res. 36, 1907-1918.

Lin, Z., and He, S. (2009). Seeing the invisible: the scope and limits of unconscious processing in binocular rivalry. Prog. Neurobiol. 87, 195-211.

Lorber, M., Zuber, B. L., and Stark, L. (1965). Suppression of the pupillary light reflex in binocular rivalry and saccadic suppression. Nature 208, 558-560.

Macmillan, N. A., and Creelman, C. D. (1991). Detection Theory: A User's Guide. New York: Cambridge University Press.

Mudrik, L., Breska, A., Lamy, D., and Deouell, L. Y. (2011). Integration without awareness: expanding the limits of unconscious processing. Psychol. Sci. 22, 764-770.

Nachmias, J., and Weber, A. (1975). Discrimination of simple and complex gratings. Vision Res. 15, 217-223.

Nguyen, V. A., Freeman, A. W., and Wenderoth, P. (2001). The depth and selectivity of suppression in binoc- ular rivalry. Percept. Psychophys. 63, 348-360.

O'Shea, R. P., and Blake, R. (1986). Dichoptic temporal frequency differences do not lead to binocular rivalry. Percept. Psychophys. 39, 59-63.

Pelli, D. G. (1997). The VideoToolbox software for visual psychophysics: transforming numbers into movies. Spat. Vis. 10, 437-442.

Polonsky, A., Blake, R., Braun, J., and Heeger, D. J. (2000). Neuronal activity in human primary visual cortex correlates with perception during binocular rivalry. Nat. Neurosci .3, 1153-1159.

Robson, J. G. (1966). Spatial and temporal contrast-sensitivity functions of the visual system. J. Opt. Soc. Am. $56,1141-1142$.

Sabrin, H. W., and Kertesz, A. E. ments and binocular rivalry. Percept. Psychophys. 28, 150-154.

Sterzer, P., Haynes, J. D., and Rees, G. (2008). Fine-scale activity patterns in high-level visual areas encode the category of invisible objects. J. Vis. 8, $1-12$.

Sterzer, P., Kleinschmidt, A., and Rees, G. (2009). The neural bases of multistable perception. Trends Cogn. Sci. (Regul. Ed.) 13, 310-318.

Tong, F., Meng, M., and Blake, R. (2006). Neural bases of binocular rivalry. Trends Cogn. Sci. (Regul. Ed.) 10, 502-511.

Tsuchiya, N., and Koch, C. (2005). Continuous flash suppression reduces negative afterimages. Nat. Neurosci. 8, 1096-1101. (1980). Microsaccadic eye move- 
van Ee, R. (2009). Stochastic variations in sensory awareness are driven by noisy neuronal adaptation: evidence from serial correlations in perceptual bistability. J. Opt. Soc. Am. A Opt. Image Sci. Vis. 26, 2612-2622.

Wade, N. J. (1974). The effect of orientation in binocular contour rivalry of real images and afterimages. Percept. Psychophys. 15, 227-232.

Watson, A. B., and Robson, J. G. (1981). Discrimination at threshold: labeled detectors in human vision. Vision Res. 21, 1115-1122.

Weiskrantz, L. (1990). The Ferrier Lecture, 1989. Outlooks for blindsight: explicit methodologies for implicit processes. Proc. R. Soc. Lond. B Biol. Sci. 239, 247-278.

Wichmann, F. A., and Hill, N. J. (2001). The psychometric function: I. Fitting, sampling, and goodness of fit. Percept. Psychophys. 63, 1293-1313.
Conflict of Interest Statement: The authors declare that the research was conducted in the absence of any commercial or financial relationships that could be construed as a potential conflict of interest.

Received: 30 July 2011; accepted: 24 October 2011; published online: 14 November 2011.

Citation: Zadbood A, Lee S$H$ and Blake $R$ (2011) Stimulus fractionation by interocular suppression. Front. Hum. Neurosci. 5:135. doi: 10.3389/fnhum.2011.00135

Copyright (c) 2011 Zadbood, Lee and Blake. This is an open-access article subject to a non-exclusive license between the authors and Frontiers Media SA, which permits use, distribution and reproduction in other forums, provided the original authors and source are credited and other Frontiers conditions are complied with. 